\title{
Two case reports of successful anesthetic management of arnold-chiari malformation type I patients who presented for elective cesarean section
}

\begin{abstract}
Optimal anesthetic care for parturients with Arnold-Chiari Malformation Type 1 (ACM-1) has been an ongoing discussion among physicians, but so far, no consensus has been reached.1 In this report, we present the successful management of two ACM-1 parturients who presented to our institution for cesarean delivery. Both general and epidural anesthesia provided effective pain relief to our parturients. A multidisciplinary approach and individualized care plans were necessary for favorable fetal and maternal outcomes.
\end{abstract}

Keywords: cesarean section, arnold-chiari malformation type 1, general anesthesia, epidural anesthesia
Volume 8 Issue 4 - 2018

\section{Levin, S Cohen, S Mellender, G Kiss}

Department of Anesthesiology, Rutgers - Robert Wood Johnson

Medical School, USA

Correspondence: Danielle Levin, Department of Anesthesiology, Rutgers - Robert Wood Johnson Medical School, USA, Email dI5I0@rwjms.rutgers.edu

Received: July 23, 2018 | Published: August 14, 2018

\section{Introduction}

The proper clinical management of parturients with Arnold-Chiari Malformation Type 1 (ACM-1) has been an ongoing discussion among physicians. Studies have been conducted to assess the optimal anesthetic care for these patients during labor and delivery, but so far, no consensus has been reached1. In this report, we present the successful management of two ACM-1 parturients who presented to our institution for cesarean delivery. Written informed consent was obtained from both patients for publication of this manuscript.

\section{Case I}

A 23 year old parturient (height 5'7', weight 200 lbs.), G2P0010, with known ACM-1, presented at 39weeks gestation with rupture of membranes. The patient's past surgical history was significant for dilation and curettage for spontaneous abortion. She was diagnosed with ACM-1 when she was 19 years old. As part of her prenatal care, the patient underwent a neurosurgical evaluation which included an MRI showing a stable Type-1 Arnold-Chiari Malformation with cerebellar tonsils occupying the foramen magnum, with compression on the medulla. Due to strong neurological opinion that an accidental dural puncture which leads to lower spinal CSF pressure due to CSF leak during epidural anesthesia, which worsen the herniation of the tonsils, outweigh the risks of transient increase in intracranial pressure that may accompany general anesthesia. Therefore, the decision was made to proceed with general anesthesia for elective cesarean section.

On the day of surgery, the patient's vital signs were BP $136 / 87$, HR 89 , temperature $98^{\circ} \mathrm{F}$. Labs were: WBC 10K, Hgb $12 \mathrm{mg} \%$, Hct 39 and platelet count $148 \mathrm{~K}$. The patient reported an allergy to latex. Prior to induction of general anesthesia, the patient received metoclopramide $10 \mathrm{mg}$ IV and citric acid/sodium citrate $30 \mathrm{~mL}$ PO. Rapid Sequence anesthesia induction was carried out using an 18-guage IV in the right arm. Lidocaine $100 \mathrm{mg}$ was injected, followed by propofol $200 \mathrm{mg}$. Succinylcholine $100 \mathrm{mg}$ was then injected and a size 7 cuffed ETT was placed and secured at depth of $21 \mathrm{~cm}$ using direct laryngoscopy with MAC 3 blade. General anesthesia was maintained using isoflurane $0.9 \% \mathrm{O}_{2}$ at $2 \mathrm{~L}$ and $\mathrm{N}_{2} \mathrm{O}$ at $2 \mathrm{~L}$. Following an uneventful delivery, the patient received $100 \mathrm{mcg}$ of fentanyl, $2 \mathrm{mg}$ of midazolam, $4 \mathrm{mg}$ of ondansetron, and 20 units of oxytocin intraoperatively. The patient remained hemodynamically stable throughout the procedure. A live female infant (Apgar score 9 and 9 at 1 and 5 minutes, respectively) was delivered. The patient was successfully extubated and transported to recovery room. The patient remained hospitalized for a total of 5 days and remained stable following surgery. Post-operative pain was well controlled using IV-PCA hydromorphone. The patient did not report any new neurological symptoms following surgery. Mother and child were discharged in stable condition on post-op day 5 .

\section{Case 2}

In this case, we present the safe use of epidural anesthesia for a cesarean section in a 30 year old, primigravid parturient at 38 weeks gestation (height 5'3", weight $173 \mathrm{lbs}$.), with known history of ACM-1 and Syringomyelia who presented with spontaneous rupture of membranes. At age 16, patient was diagnosed with ACM1 with MRI significant for descent of the cerebellar tonsils $8 \mathrm{~mm}$ on the right and $6 \mathrm{~mm}$ on the left below the level of the foramen magnum. A Syringomyelia cyst was also identified extending from C6 to T1. Patient's condition since diagnosis has been unchanged and remarkably asymptomatic with infrequent migraine-type headaches. She denied headaches with postural changes, incoordination, numbness, weakness or any other focal neurological deficits. Course of pregnancy was uncomplicated with appropriate prenatal care. Physical exam was unremarkable. Labs were: $\mathrm{Hgb} 14.2 \mathrm{mcg} \%$, Hct 40.1, WBC $9.9 \mathrm{~K}$, and platelet count $208 \mathrm{~K}$.

After consultation with her obstetrician and neurologist, the decision to pursue an elective caesarean section was made to avoid increased ICP during active labor, which would increase CSF flow into the syrinx and increase the pressure on the distended spinal cord inside a fixed sized spinal canal. Epidural anesthesia with the application of our gravity technique ${ }^{2}$ was considered because it could provide less changes in epidural pressure during administration of local anesthetics. The risks and benefits of this procedure were discussed with the patient and after informed consent, an epidural was performed using aseptic technique while the patient was awake and in left lateral decubitus position. 
The L3-L4 level was identified, and a skin wheal was made at the needle entry site. The overlying skin and subcutaneous tissue were appropriately anesthetized. A 17-gauge epidural needle was advanced at the midline into the lumbar epidural space at L3-4 using the lossof-resistance to air technique. The patient received a total of $20 \mathrm{ml}$ of $2 \%$ lidocaine with an adjunct of fentanyl $5 \mathrm{mcg} / \mathrm{ml}$ and epinephrine $5 \mathrm{mcg} / \mathrm{ml}$, applying a low-pressure gravity administration technique via IV extension tubing. The local anesthetic administration was made incrementally with constant monitoring every 5 minutes allowing significantly low pressure administration. The epidural technique was well tolerated without any change in vital or neurological signs. A low transverse caesarean section was performed and a live male infant (Apgar 9/9) was delivered without complications. The parturient was transferred to post-anesthesia care units where she then received epidural PCA ropivacaine $0.025 \%$ with fentanyl $3 \mathrm{mcg} / \mathrm{ml} 15 \mathrm{ml} / \mathrm{hr}$, PCA dose $4 \mathrm{ml}$, and lockout time 10 minutes for 48 hours. Patient remained stable during hospital stay and at discharge on post-op day 3.

\section{Discussion}

Arnold-Chiari Malformation Type 1 (ACM-1) is a congenital malformation characterized by herniation of the cerebellar tonsils below the level of the foramen magnum. ${ }^{3}$ In patients with type I ACM, any potential cause of increased intracranial pressure must be avoided as it can result in the transfer of more spinal fluid into the Syringomyelia cyst. This increases the size of the spinal cord inside a fixed spinal canal size along with further spinal cord compression with rapid deterioration of neurological function and further herniation of the cerebellar tonsils. ${ }^{4}$ Careful consideration is necessary to determine the best method of delivery and optimal anesthetic delivery so that the risk of potential complications secondary to changes in cerebrospinal fluid (CSF) pressure is minimized.

Uterine contractions and bearing down during vaginal labor have been reported to increase CSF pressure ${ }^{5,6}$ which increases spinal cord size. Upon consultation with the parturients' neurologists and obstetricians, cesarean section was selected as the delivery method in both cases in order to avoid more compression on the spinal cord and more tonsillar herniation. A retrospective case series of 12 patients suggested that regional and general anesthesia were both safe and effective choices in these patients. ${ }^{7}$ However, general anesthesia presents the risk of elevated CSF pressure and increase in ICP due to coughing and bucking, especially during induction and emergence of a potentially difficult intubation (BMI $>30)$. On the other hand, epidural anesthesia presents the potential risk of an accidental dural puncture resulting in a sudden reduction of CSF pressure, leading to risk of further herniation ${ }^{8}$ and the potential for postdural puncture headache.

In our first case, we demonstrated the relative safety of using general inhalational anesthesia with rapid sequence induction in pregnant patients with ACM-1 during caesarean section in order to minimize possible complications accompanying reduction in CSF pressure from spinal or epidural anesthesia that can occur with accidental dural puncture. Decrease in CSF pressure can cause further herniation of the cerebellar tonsils.
The application of epidural anesthetics for the second case had no neurological deficits probably due to fewer fluctuations in CSF pressure. To minimize the risk of accidental dural puncture, we positioned the patient in the lateral decubital position, administered the epidural anesthesia using the gravity technique 2 , and reinserted the stylet any time the needle was advanced. ${ }^{9}$ The epidural anesthesia was successfully administered without complications. In this case, we demonstrated the safe use of epidural anesthesia for a cesarean section in a patient with minimally symptomatic Type I Arnold-Chiari Malformation and Syringomyelia without tonsillar herniation.

In summary, it is important to tailor the treatment of our parturients with ACM-1 who present for labor and delivery to their specific medical conditions. Both general anesthesia and epidural anesthesia can provide safe and effective pain relief to parturients with ACM-1. A multidisciplinary approach of anesthesiologists, neurologists, and obstetricians should be used to choose the best clinical care for the parturient. We emphasize the importance of individualized care plans for favorable fetal and maternal outcomes.

\section{Acknowledgments}

None.

\section{Conflicts of Interest}

The author declaresno conflicts of interest.

\section{References}

1. Ghaly RF, Tverdohleb T, Candido KD, et al. Management of parturients in active labor with Arnold Chiari malformation, tonsillar herniation, and syringomyelia. Surg Neurol Int. 2017;8:10.

2. Cohen S, Amar D. Epidural block for obstetrics: Comparison of bolus injection of local anesthetic with gravity flow technique. J Clin Anesth. 9(8):623-628.

3. Vannemreddy P, Nourbakhsh A, Willis B, et al. Congenital Chiari malformations. Neurol India. 2010;58(1):6-14.

4. Dyste GN, Menezes AH, Van Gilder JC. Symptomatic Chiari malformations. An analysis of presentation, management, and long-term outcome. J Neurosurg. 1989;71(2):159-168.

5. McCausland AM, Holmes F. Spinal fluid pressures during labor; preliminary report. West J Surg Obstet Gynecol. 1957;65(4):220-231.

6. Marx GF, Oka Y, Orkin LR. Cerebrospinal fluid pressures during labor. $A m$ J Obstet Gynecol. 1962;84:213-219.

7. Chantigian RC, Koehn MA, Ramin KD, et al. Chiari I malformation in parturients. J Clin Anesth. 2002;14(3):201-205.

8. Hullander RM, Bogard TD, Leivers D, et al. Chiari I malformation presenting as recurrent spinal headache. Anesth Analg. 1992;75(6):10251026 .

9. Cohen S, Maestrado P, Zada Y. A simple technique to reduce the incidence of accidental dural puncture. Anaesthesia. 2001;56(7):690-713. 